\title{
A New Block Markov Coding Strategy for Pairwise and Collective Cooperation in the Three User MAC
}

\author{
Çag̃atay Edemen and Onur Kaya \\ Department of Electrical and Electronics Engineering \\ Isik University, Istanbul, Turkey \\ Email: \{cagatayedemen,onurkaya\}@isikun.edu.tr
}

\begin{abstract}
For a three user cooperative multiple access channel (MAC), we propose a new block Markov superposition encoding strategy, which enables all three users to cooperate collectively as well as in pairs. We obtain the resulting achievable rate expressions and compare them with existing two and three user cooperative strategies. We demonstrate that significant rate gains may be possible, without resorting to adaptive encoding/decoding techniques. We investigate the contributions from pairwise and collective cooperation signals while achieving tuples on the rate region boundary, and compare by simulations the sum rates achievable by two user versus three user grouping in cooperative MACs with fixed total resources.
\end{abstract}

\section{INTRODUCTION}

Roots of user cooperation date back to the introduction of the relay channel by van der Meulen [1]. This channel was investigated extensively in [2], where a number of relaying strategies, achievable rates and upper bounds on the capacity of a general relay channel were obtained. Ever since, relay channels have been the subject of an extensive amount of work in the literature, a thorough review of which can be found in [3].

The concept of relaying is certainly not limited to a single source-single relay scenario, and there has also been significant research on relaying in larger networks. Among multi-user relay models are the parallel relay network (PRN) [4], the multiple relay network (MRN) [3], [5], the multiple access relay channel (MARC) [6], and the broadcast relay channel [7]. All of these models can be considered as forms of user cooperation, but are limited in the sense that they all involve dedicated relays not capable of transmitting their own information.

A fairer cooperation scenario is one in which all communicating transmitters have their own messages, and mutually cooperate in their transmissions. One approach to multi-user cooperation is that transmitters use orthogonal dimensions (time/frequency) and take turns in relaying each other's messages [8]; but this approach has the downside that it requires careful medium access control, and suffers from rate loss due to orthogonality. An alternative abstract model which allows for simultaneous mutual cooperation, MAC with generalized feedback, was first studied by Carleial [9], and then by Willems et al. [10]. Sendonaris et al. [11] applied the results of [10] to obtain the achievable rates for a two user cooperative Gaussian MAC in the presence of fading, and obtained a remarkable improvement in the achievable rates, over the

This work was supported by TUBITAK Grant 111E108. traditional MAC with non-cooperating transmitters. However, these works all deal with a two-user cooperative MAC.

An attempt to generalize the results of [10], [11] to more than two users was made in [12], [13]. There, we have introduced the three user MAC with generalized feedback model which contains MARC, MRN and PRN with three users and one receiver as its special cases, and obtained achievable rate regions based on three user channel adaptive block Markov encoding (BME) and backwards decoding. The proposed BME strategy assigned varying roles to users in cooperation: users with stronger receive links decoded more cooperative signals, and therefore participated in cooperative transmissions more actively. Although this non-trivial extension of BME was shown to produce considerable rate gains, it has the drawback that it requires instantaneous adaptation of encoding/decoding policies, which increases system complexity. A MIMO extension of the three user cooperative MAC was considered more recently in [14], but there either conferencing encoders were assumed, or the common messages among the users were assumed to be already established, thereby not requiring any resources, and not causing any constraints for inter-user transmissions. In this paper, we propose a novel non-adaptive three user BME strategy to simultaneously establish and send common information. In our encoding model, each user divides its message into submessages, each of which is dedicated for either pairwise or collective (three user) cooperation, but not both. Using this strategy, we obtain a set of achievable rates for the three user cooperative MAC, and show that this set not only improves upon two user cooperation or MARC significantly, but it may even outperform the adaptive BME strategy, when the cooperative links are much stronger than direct links to the receiver.

\section{SYSTEM MODEL}

We consider a fading three user cooperative Gaussian MAC with full duplex operation, modeled by

$$
\begin{aligned}
& Y_{0}=\sqrt{s_{10}} X_{1}+\sqrt{s_{20}} X_{2}+\sqrt{s_{30}} X_{3}+N_{0} \\
& Y_{1}=\sqrt{s_{21}} X_{2}+\sqrt{s_{31}} X_{3}+N_{1} \\
& Y_{2}=\sqrt{s_{12}} X_{1}+\sqrt{s_{32}} X_{3}+N_{2} \\
& Y_{3}=\sqrt{s_{13}} X_{1}+\sqrt{s_{23}} X_{2}+N_{3}
\end{aligned}
$$

where $X_{i}$ is the symbol transmitted by node $i$, satisfying $E\left[X_{i}^{2}\right] \leq P_{i}, Y_{j}$ is the effective received symbol at node $j$ after subtraction of any self interference, and the receiver is denoted by $j=0 ; N_{j} \sim \mathcal{N}(0,1)$ is the white Gaussian noise 


$$
\begin{aligned}
\sum_{l \in \Gamma_{1}^{(i)}} R_{l i}+\sum_{m \in \Gamma_{2}^{(i)}} R_{m U}<E\left[C\left(\sum_{l \in \Gamma_{1}^{(i)}} \frac{s_{l i} P_{l i}}{I_{i}}+\sum_{m \in \Gamma_{2}^{(i)}} \frac{s_{m i} P_{X_{m U}}}{I_{i}}\right)\right] \forall i \in S, \forall \Gamma_{1}^{(i)} \subset\{i\}^{c}, \forall \Gamma_{2}^{(i)} \subset\{i\}^{c} \\
\sum_{\{i, j\} \in \Gamma_{3}} R_{i j}+R_{j i}<E\left[C\left(\sum_{\{i, j\} \in \Gamma_{3}}\left(s_{i 0}\left(P_{i j}+P_{U_{i j}}\right)+s_{j 0}\left(P_{j i}+P_{U_{j i}}\right)+2 \sqrt{s_{i 0} s_{j 0} P_{U_{i j}} P_{U_{j i}}}\right)\right)\right], \quad \forall \Gamma_{3} \subset \Gamma \\
R_{1}+R_{2}+R_{3}<E\left[C\left(s_{10} P_{1}+s_{20} P_{2}+s_{30} P_{3}+\sum_{\{i, j\} \in \Gamma}\left(2 \sqrt{s_{i 0} s_{j 0} P_{U_{i j}} P_{U_{j i}}}+2 \sqrt{s_{i 0} s_{j 0} P_{U_{i}} P_{U_{j}}}\right)\right)\right]
\end{aligned}
$$

at node $j$, and $\sqrt{s_{i j}}$ are the normalized (for unit noise power) Rayleigh distributed fading coefficients with parameters $\gamma_{i j}$, the realizations of which are assumed to be known by both the transmitters and the receiver. A few words on the notation used throughout this paper: from now on, we use the user indices $i \neq j \neq k$ to denote distinct elements of the set $S \triangleq\{1,2,3\}$; hence, when $i$ and $j$ are fixed, $k=\{i, j\}^{c}$, where $\{\cdot\}^{c}$ denotes the set complement operation. The set $\Gamma=$ $\{\{1,2\},\{2,3\},\{3,1\}\}$ is used frequently while describing the encoding strategy and achievable rates. Random variables are denoted by capital letters $X$, while their realizations are denoted by their lowercase versions $x$.

\section{Achievable Rates via Three User BME}

Let $R_{i}$ denote the rate of user $i$, in bits per transmission. Our main achievability result is presented in the following theorem.

Theorem 1: For the three user cooperative MAC, define the rate triplet $\left(R_{1}, R_{2}, R_{3}\right)$, with $R_{i}=R_{i j}+R_{i k}+R_{i U}, \forall i \neq j \neq$ $k \in S$. Then, an achievable rate region is given by the closure of the convex hull of all rate triplets $\left(R_{1}, R_{2}, R_{3}\right)$ satisfying (5)-(7) at the top of this page, where $C(x) \triangleq \frac{1}{2} \log _{2}(1+x)$;

$$
I_{i}=1+s_{j i}\left(P_{j k}+P_{U_{j k}}\right)+s_{k i}\left(P_{k j}+P_{U_{k j}}\right)+2 \sqrt{s_{j i} s_{k i} P_{U_{j k}} P_{U_{k j}}}
$$

denotes the interference at user $i$ and the convex hull is taken over all power allocation policies that obey

$$
P_{i j}+P_{i k}+P_{X_{i U}}+P_{U_{i j}}+P_{U_{i k}}+P_{U_{i}} \leq P_{i}, \quad \forall i \in S
$$

where the variables on the LHS of (8) are the powers assigned to individual component codewords, used in encoding.

Proof: (Outline) The proof, as in most information theoretical achievability results, consists of message generation, codebook generation, encoding and decoding policies, followed by probability of error analysis. Here, we provide an outline of the proof, highlighting the novel points specific to this work, and refer the more standard error probability analysis to the literature.

Message Generation: To establish both pairwise and collective cooperation among the users, the message $w_{i}(b)$ of each user $i \in S$, in each transmission block $b \in\{1, \ldots, B\}$, is divided into three independent submessages $w_{i j}(b), w_{i k}(b)$ and $w_{i U}(b)$, taking values from index sets $\mathcal{W}_{i j} \triangleq\left\{1, \cdots, 2^{n R_{i j}}\right\}$, $\mathcal{W}_{i k} \triangleq\left\{1, \cdots, 2^{n R_{i k}}\right\}$ and $\mathcal{W}_{i U} \triangleq\left\{1, \cdots, 2^{n R_{i U}}\right\}$ respectively. Messages $w_{i j}(b)$ and $w_{i k}(b)$ are intended to be decoded at users $j$ and $k$ respectively, to facilitate pairwise cooperation in the next block; and $w_{i U}(b)$, is to be decoded by both user $j$ and user $k$, to facilitate collective cooperation ${ }^{1}$.

Codebook Generation: Fix a distribution

$$
p(z)=p(u) \prod_{\{i, j\} \in \Gamma} p\left(u_{i j} \mid u\right) p\left(x_{i U} \mid u\right) p\left(x_{i j} \mid u, u_{i j}\right) p\left(x_{i k} \mid u, u_{i k}\right)
$$

where $Z=\left\{U, U_{i j}, X_{i U}, X_{i j}, X_{i k},\{i, j\} \in \Gamma\right\}$, is a joint random variable, and $U_{i j}=U_{j i}, \forall\{i, j\} \in \Gamma$. Let all distributions on the right hand side of (9) be unit Gaussian. Then, the codebook generation proceeds as follows:

- Generate $2^{n\left(R_{1 U}+R_{2 U}+R_{3 U}\right)}$ sequences $\mathbf{U}^{n}$, from the distribution $p\left(\mathbf{u}^{n}\right)=\prod_{l=1}^{n} p\left(u_{l}\right)$. Assign each sequence to a distinct message triplet $\left\{w_{1 U}^{\prime}, w_{2 U}^{\prime}, w_{3 U}^{\prime}\right\} \in \mathcal{W}_{1 U} \times \mathcal{W}_{2 U} \times \mathcal{W}_{3 U}$, and call them $\mathbf{u}^{n}\left(w_{1 U}^{\prime}, w_{2 U}^{\prime}, w_{3 U}^{\prime}\right)$.

- For every $\{i, j\} \in \Gamma$, and every $\mathbf{u}^{n}\left(w_{1 U}^{\prime}, w_{2 U}^{\prime}, w_{3 U}^{\prime}\right)$, generate $2^{n\left(R_{i j}+R_{j i}\right)}$ sequences $\mathbf{U}_{i j}^{n}$, from the distribution $p\left(\mathbf{u}_{i j}^{n} \mid \mathbf{u}^{n}\right)=\prod_{l=1}^{n} p\left(u_{i j l} \mid u_{l}\right)$. Assign each sequence to a distinct message pair $\left\{w_{i j}^{\prime}, w_{j i}^{\prime}\right\} \in \mathcal{W}_{i j} \times \mathcal{W}_{j i}$, and call them $\mathbf{u}_{i j}^{n}\left(w_{i j}^{\prime}, w_{j i}^{\prime}, \mathbf{u}^{n}\right)$. Let $\mathbf{u}_{j i}^{n}=\mathbf{u}_{i j}^{n}$.

- For every $i \in S$, and every $\mathbf{u}^{n}\left(w_{1 U}^{\prime}, w_{2 U}^{\prime}, w_{3 U}^{\prime}\right)$, generate $2^{n R_{i U}}$ sequences $\mathbf{X}_{i U}^{n}$, from the distribution $p\left(\mathbf{x}_{i U}^{n} \mid \mathbf{u}^{n}\right)=$ $\prod_{l=1}^{n} p\left(x_{i U l} \mid u_{l}\right)$. Assign each sequence to a distinct message $w_{i U} \in \mathcal{W}_{i U}$, and call them $\mathbf{x}_{i U}^{n}\left(w_{i U}, \mathbf{u}^{n}\right)$.

- For every $\{i, j\} \in \Gamma$, and for every pair $\mathbf{u}^{n}\left(w_{1 U}^{\prime}, w_{2 U}^{\prime}, w_{3 U}^{\prime}\right)$ and $\mathbf{u}_{i j}^{n}\left(w_{i j}^{\prime}, w_{j i}^{\prime}, \mathbf{u}^{n}\right)$, generate $2^{n R_{i j}}$ sequences $\mathbf{X}_{i j}^{n}$, from the distribution $p\left(\mathbf{x}_{i j}^{n} \mid \mathbf{u}^{n}, \mathbf{u}_{i j}^{n}\right)=\prod_{l=1}^{n} p\left(x_{i j l} \mid u_{l}, u_{i j l}\right)$. Assign each sequence to a distinct message $w_{i j} \in \mathcal{W}_{i j}$, and call them $\mathbf{x}_{i j}^{n}\left(w_{i j}, \mathbf{u}^{n}, \mathbf{u}_{i j}^{n}\right)$. Also, for the same pair $\{i, j\}$, (and hence $\left.k=\{i, j\}^{c}\right)$, for every $\mathbf{u}^{n}\left(w_{1 U}^{\prime}, w_{2 U}^{\prime}, w_{3 U}^{\prime}\right)$, and $\mathbf{u}_{i k}^{n}\left(w_{i k}^{\prime}, w_{k i}^{\prime}, \mathbf{u}^{n}\right)$, generate $2^{n R_{i k}}$ sequences $\mathbf{X}_{i k}^{n}$, from the distribution $p\left(\mathbf{x}_{i k}^{n} \mid \mathbf{u}^{n}, \mathbf{u}_{i k}^{n}\right)=\prod_{l=1}^{n} p\left(x_{i k l} \mid u_{l}, u_{i k l}\right)$. Assign each sequence to a distinct message $w_{i k} \in \mathcal{W}_{i k}$, and call them $\mathbf{x}_{i k}^{n}\left(w_{i k}, \mathbf{u}^{n}, \mathbf{u}_{i k}^{n}\right)$.

Encoding: At the beginning of each transmission block $b$, each user $i$ has the messages $w_{j i}^{\prime} \triangleq w_{j i}(b-1), w_{k i}^{\prime} \triangleq$ $w_{k i}(b-1), w_{j U}^{\prime} \triangleq w_{j U}(b-1)$ and $w_{k U}^{\prime} \triangleq w_{k U}(b-1)$ it has correctly decoded in the previous block (guaranteed by the rate constraints (5), which will be discussed in decoding

${ }^{1}$ It is also possible to add a fourth submessage $w_{i 0}(b)$, intended to be decoded solely at the receiver, and the encoding policy can be modified accordingly. In this case, the achievable rate expressions become rather long and complicated; and are therefore relegated to the journal version. 


\begin{tabular}{ll} 
Codeword $($ User $i)$ & Task \\
\hline \hline$X_{i j}\left(w_{i j}(b), U_{i j}(b), U(b)\right)$ & create common information $w_{i j}(b)$ with user $j$, help decode $U_{i j}(b)$ and $U(b)$ at the receiver. \\
$X_{i k}\left(w_{i k}(b), U_{i k}(b), U(b)\right)$ & create common information $w_{i k}(b)$ with user $k$, help decode $U_{i k}(b)$ and $U(b)$ at the receiver. \\
$X_{i U}\left(w_{i U}(b), U(b)\right)$ & create common information $w_{i U}(b)$ with users $j$ and $k$, help decode $U(b)$ at the receiver. \\
$U_{i j}(b) \triangleq U_{i j}\left(w_{i j}(b-1), w_{j i}(b-1), U(b)\right)$ & cooperate with $j$ to transmit $w_{i j}(b-1)$ and $w_{j i}(b-1)$ to receiver, help decode $U(b)$ at the receiver. \\
$U_{i k}(b) \triangleq U_{i k}\left(w_{i k}(b-1), w_{k i}(b-1), U(b)\right)$ & cooperate with $k$ to transmit $w_{i k}(b-1)$ and $w_{k i}(b-1)$ to receiver, help decode $U(b)$ at the receiver. \\
$U(b) \triangleq U\left(w_{i U}(b-1), w_{j U}(b-1), w_{k U}(b-1)\right)$ & collectively cooperate with $j$ and $k$, enable a triple coherent combining gain while decoding common messages. \\
\hline
\end{tabular}

TABLE II: Codebook generation and BME.

\begin{tabular}{lll}
\hline & DECODED MESSAGES & OWN MESSAGES \\
\hline \hline USER I & $w_{21}^{\prime}, w_{31}^{\prime}, w_{2 U}^{\prime}, w_{3 U}^{\prime}$ & $w_{12}, w_{13}, w_{1 U}$ \\
USER II & $w_{12}^{\prime}, w_{32}^{\prime}, w_{1 U}^{\prime}, w_{3 U}^{\prime}$ & $w_{21}, w_{23}, w_{2 U}$ \\
USER III & $w_{13}^{\prime}, w_{23}^{\prime}, w_{1 U}^{\prime}, w_{2 U}^{\prime}$ & $w_{31}, w_{32}, w_{3 U}$ \\
\hline
\end{tabular}

TABLE I: List of messages known to each user in block $b$.

section), as well as its own messages, as summarized in Table I. Each user then selects the codewords corresponding to these messages, from the generated codebooks, as shown in Table II. Finally, the overall transmitted codeword of each user $i \in S$, is obtained by scaling the codewords listed in Table II to have the desired power levels, and superposing them, i.e.,

$$
\begin{aligned}
X_{i}= & \sqrt{P_{i j}} X_{i j}+\sqrt{P_{i k}} X_{i k}+\sqrt{P_{X_{i U}}} X_{i U} \\
& +\sqrt{P_{U_{i j}}} U_{i j}+\sqrt{P_{U_{i k}}} U_{i k}+\sqrt{P_{U_{i}}} U,
\end{aligned}
$$

yielding our proposed three user BME strategy. Note that, the BME strategy employs two main types of codewords: $X_{i j}$, $X_{i k}$ and $X_{i U}$, which are used to transmit fresh information at a higher data rate than normally decodable by the receiver, and $U_{i j}, U_{i k}$, and $U$, which are cooperatively used to resolve the receiver's remaining uncertainty from previous transmissions. In Table II, the specific tasks of these codewords are also explained.

Decoding: The decoding at the users (transmitters) is executed at the end of each block, based on joint typicality check. The channel towards each user $i$ may be viewed as a two user MAC, where users $j$ and $k$ transmit several independent messages, but user $i$ only decodes the messages $w_{j i}, w_{k i}$, $w_{j U}$ and $w_{k U}$, while treating codewords devoted to other messages, namely $X_{j k}, X_{k j}$ and $U_{j k}=U_{k j}$ as noise. Note that, assuming the previous decoding stages were error free, user $i$ already knows the codewords $U_{i j}=U_{j i}, U_{i k}=U_{k i}$, $U$ and $X_{i}$. Therefore, user $i$ searches for $w_{j i}, w_{k i}, w_{j U}$ and $w_{k U}$, that make $\left\{X_{j i}, X_{k i}, X_{j U}, X_{k U}, Y_{i}\right\}$ jointly typical, given $U_{i j}=U_{j i}, U_{i k}=U_{k i}, U$ and $X_{i}$. Then, using traditional results on the capacity of a MAC [16], it is straightforward to show that the probability of user $i$ decoding the messages listed above incorrectly goes to zero, if the rates of these messages satisfy

$$
\begin{aligned}
& \sum_{l \in \Gamma_{1}^{(i)}} R_{l i}+\sum_{m \in \Gamma_{2}^{(i)}} R_{m U}<I\left(X\left(\Gamma_{1}^{(i)}\right), X\left(\Gamma_{2}^{(i)}\right) ; Y_{i} \mid X_{i}, U, U_{i j},\right. \\
&\left.U_{i k}, X\left(\left\{\Gamma_{1}^{(i)}\right\}^{c}\right), X\left(\left\{\Gamma_{2}^{(i)}\right\}^{c}, \mathbf{s}\right)\right) \\
& \forall \Gamma_{1}^{(i)} \subset\{i\}^{c}, \forall \Gamma_{2}^{(i)} \subset\{i\}^{c}
\end{aligned}
$$

where $\mathbf{s}$ denotes the vector of channel states, $X\left(\Gamma_{1}^{(i)}\right) \triangleq$ $\left\{X_{l i}: l \in \Gamma_{1}^{(i)}\right\}$ and $X\left(\Gamma_{2}^{(i)}\right) \triangleq\left\{X_{m U}: m \in \Gamma_{2}^{(i)}\right\}$ denote codeword vector sets, some potentially empty. This results in 15 equations per user. Evaluating for Gaussian codewords, we obtain (5).

The decoding at the receiver is performed after all $B$ blocks of information are received, using backwards decoding. As commonly done in BME, no fresh information is transmitted in block $B$, hence the codewords $X_{i j}, X_{j i}, U_{i j}$ and $U_{j i}$ are all used to decode the pair $\left\{w_{i j}(B-1), w_{j i}(B-1)\right\}$, for each $\{i, j\} \in \Gamma$. Similarly, $w_{i U}(B-1), w_{j U}(B-1)$ and $w_{k U}(B-1)$ are also decoded in the last block, by using all of the received codewords, as each codeword is also a function of $U$. Since the messages are jointly decoded, asymptotically error free decoding is possible, following similar lines to the two user case [10], if, $\forall \Gamma_{3} \subset \Gamma$,

$$
\begin{gathered}
\sum_{\{i, j\} \in \Gamma_{3}} R_{i j}+R_{j i}<I\left(X\left(\Gamma_{3}\right), X\left(\bar{\Gamma}_{3}\right), U\left(\Gamma_{3}\right), U\left(\bar{\Gamma}_{3}\right) ; Y \mid U\right. \\
\left.X\left(\Gamma_{3}^{c}\right), X\left(\bar{\Gamma}_{3}^{c}\right), U\left(\Gamma_{3}^{c}\right), U\left(\bar{\Gamma}_{3}^{c}\right), \mathbf{s}\right) \\
R_{1 U}+R_{2 U}+R_{3 U}<I\left(X_{1}, X_{2}, X_{3} ; Y \mid \mathbf{s}\right) \\
\sum_{\{i, j\} \in \Gamma_{3}} R_{i j}+R_{j i}+R_{1 U}+R_{2 U}+R_{3 U}<I\left(X_{1}, X_{2}, X_{3} ; Y \mid \mathbf{s}\right)
\end{gathered}
$$

where $\bar{\Gamma}_{3} \triangleq\left\{\{j, i\}:\{i, j\} \in \Gamma_{3}\right\}$. Once the messages indexed by $(B-1)$ are decoded, they can be used together with the codewords received in block $(B-1)$ to decode $w_{i j}(B-2)$, $w_{j i}(B-2), w_{i U}(B-2), w_{j U}(B-2)$, and $w_{k U}(B-2)$; and this process continues until all the pairwise and collective cooperative messages are correctly decoded, resulting at each step in the same rate expressions as those given in (12)-(14). Note that the codewords $X_{i j}(b), X_{i k}(b)$ and $X_{i U}(b)$ are not used at the receiver to decode the fresh information they carry, i.e., $w_{i j}(b), w_{i k}(b), w_{i U}(b)$, but are only used to aid the joint decoding of the cooperative messages from the previous block. Finally, noting that the right hand sides of (13) and (14) are identical, it is sufficient to include the strictest of these constraints, namely (14) with $\Gamma_{3}=\Gamma$, which dominates all others. Evaluating for Gaussian codewords, we obtain (6) from (12) and (7) from (14).

\section{Simulation Results}

The rate region compactly characterized in (5)-(7) is in fact governed by a total of 53 simultaneous constraints on the rates, and its simulation is a challenge in its own right. In this section, we evaluate the rate constrains under several fading scenarios, to compare our rate region to some known results, and to further investigate the usefulness of each cooperative 
codeword component in achieving rate tuples on the rate region boundary.

In Figure 1, we compare our proposed strategy to adaptive BME [13], 2-user cooperation [11], and an outer bound, which assumes co-located transmitters. The achievable rate regions are obtained under Rayleigh fading, with varying direct link and inter-user link average SNRs ( 0.5 vs $1,0.5$ vs 2 and 0.5 vs 5, respectively). For the ease of demonstration, only a slice of each of the 3-D three user rate regions, with $R_{3}=0$, (the case when user 3 acts like a relay for the other two users) are shown.

First observation, based on the innermost three regions (SNRs 0.5 vs 1 ) is that, both three user cooperative strategies expectedly surpass two user cooperation in terms of achievable rates. At these SNRs, when the direct links are moderately stronger than cooperative links, the adaptive strategy of [13] gives the largest rate region. For fading set 2 (SNRs 0.5 vs 2), the proposed strategy, although non-adaptive, performs nearly as well as the adaptive strategy near the sum rate point, and even better near the single user rates. For set 3, where the cooperative links are much stronger than the direct links (SNRs 0.5 vs 5), our proposed dedicated cooperation strategy outperforms the adaptive strategy of [13] for all rate pairs. This can be explained by a closer look at the structure of the encoding policy: the collective cooperation in our BME strategy is established through dedicated messages, $w_{i U}$, which are then mapped to the codeword $U$; whereas in [12], [13], the users decode as many of the pairwise cooperative messages as possible, even those not intended for themselves, to form the collective cooperative codewords. When the channel gains among the users are equally very strong, the latter approach puts additional unnecessary constraints on the rates at the transmitters, while for our proposed non-adaptive approach, the power distribution achieving the points on the capacity region dictates that the users should not use the pairwise cooperation signals $\left(X_{i j}\right)$, and instead they should only cooperate collectively via $X_{i U}$ and $U$. Such encoding results in looser rate constraints at the transmitters (no noise terms due to unintended messages), and overall a better rate region. Finally, we observe that the achievable rate region is not very far from the outer bound, which is obtained under the unfair assumption that all transmitters are co-located, with common information.

In Figure 2, we compare the rate regions achieved by our proposed three user cooperation strategy, with those for a two user cooperative MAC, and a two user one relay MARC [6] under several Rayleigh fading scenarios with means indicated in the figure, and equal user powers. Note that the MARC is the closest model to three user cooperative MAC: if one of the users' rate is set to 0 in our achievable rate region, so as to force dedicated relaying like in the MARC, we obtain a MARC, but with cooperating encoders. The results in Figure 2 are particularly interesting, because the comparison of our rate region with that of the MARC demonstrates the additional gain due to cooperation among the two transmitters, while being helped by a relay; whereas the comparison of our rate region with that of two user cooperation demonstrates

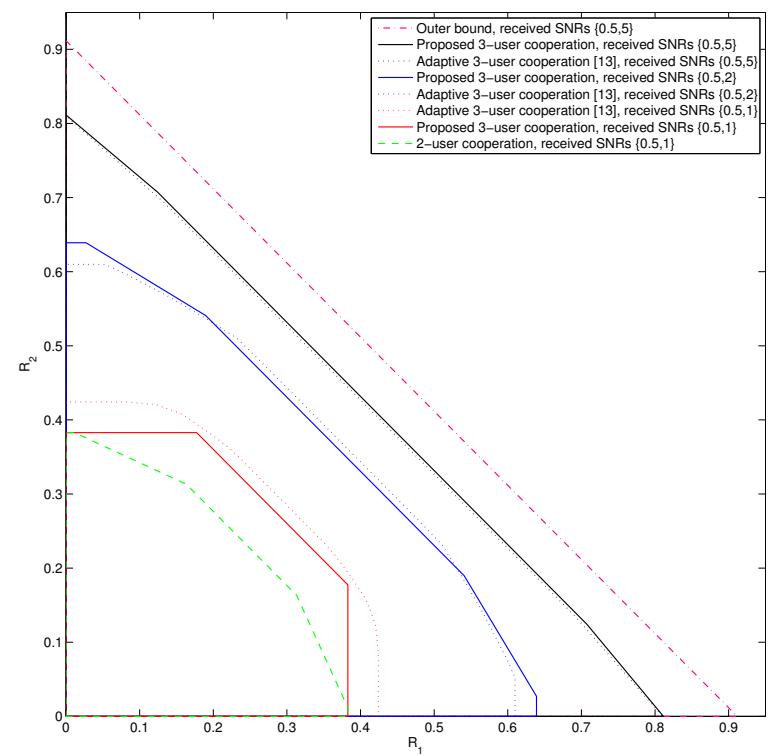

Fig. 1: Comparison of achievable rates for two user cooperation, three user cooperation with channel adaptive BME [13], and dedicated three user cooperation.

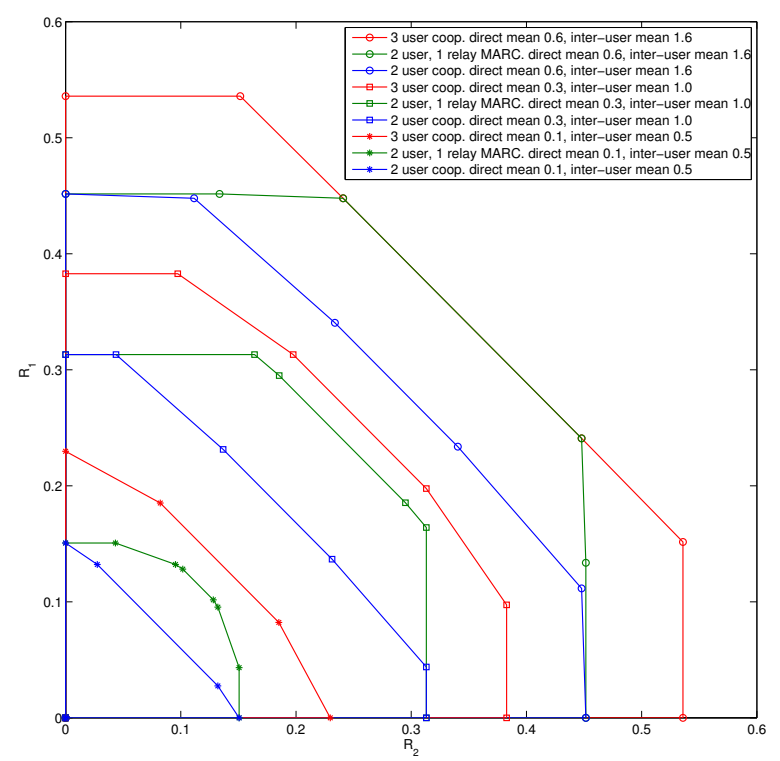

Fig. 2: Comparison of achievable rates for two user cooperation, two-user one-relay MARC, and dedicated three user cooperation.

the gain obtained by further assigning a dedicated relay to a already cooperating pair of users. We see that, the maximum individual rates $\left(R_{i}\right.$ intercepts in the figure) of the users are not improved by MARC versus two-user cooperation, as in each setup there is one dedicated relay. In contrast, three user cooperation provides an additional relay per user, hence the single user rates improve, especially when the direct link gains are relatively low, which makes cooperation more valuable. MARC gives improvement versus two user cooperation near the sum rate point, because additional relay's power can be used to relay the users' messages, while in two user cooperation, the users have to allocate some of their own power for cooperation. However, especially when the direct 
links are weak, and the inter-user links are strong, the cost of establishing common information is low, and additional three-way coherent combining gain of our proposed strategy improves the entire rate region, compared to MARC with the same resources. Note that, in three user cooperation, it is also possible to select a non-zero rate for the third user, in expense of some of the rate gains in $R_{1}$ and $R_{2}$, which is fairer than the MARC.

In Figure 3, we investigate which codewords are active while achieving several points on the rate region boundary, under symmetric and asymmetric fading. The codewords that are assigned non-zero powers are listed next to the rate tuples. In the symmetric setup (set I), collective cooperation is used throughout, and pairwise cooperation signal powers are set to zero. In the asymmetric setup (set II), the fading coefficients favor user 3 relaying messages of user 2, while user 2 relays messages of user 1 , especially near $R_{2}$ and $R_{1}$ axes respectively. Near the sum rate point, we see pairwise and collective cooperation signals are both being used; interestingly user 2 decodes message of user 1, but does not relay it, and user 3 uses the common cooperation signal $U_{3}=U_{1}=U$ to help user 1 while it uses pairwise cooperation signal $U_{32}=U_{23}$ to help user 2.

In Figure 4, we investigate the gain from three user cooperation versus two user cooperation on a fairer ground: we consider a six user setup with fixed total bandwidth and identical user powers, and consider grouping the users into two triplets versus three pairs. The channels are assumed to be symmetric, therefore it is immaterial which users go into which group. The sum rate of the system is plotted against the common mean inter-user link gain, while keeping the mean direct link gain constant. We see that except very low quality inter-user links, when the extra cooperating user causes additional interference during user-side decoding, three user cooperation with the same resources is always more beneficial.

\section{CONClusion}

We introduced a new three user block Markov encoding strategy, in which pairwise and collective cooperation is performed based on dedicated sub-messages, and obtained the resulting achievable rate regions. We demonstrated that these regions are larger than achievable rate regions for two user cooperative MAC, two user one relay MARC, and may even be larger than those for known adaptive three user encoding/decoding policies.

\section{REFERENCES}

[1] E. C. Van Der Meulen. Three-terminal communication channels. Adv. Appl. Prob., vol. 3, p. 120-154, 1971.

[2] T. M. Cover and A. El Gamal. Capacity theorems for the relay channel. IEEE Trans. Inf. Theory, vol. IT-25, no. 5, p. 572-584, Sep. 1979.

[3] G. Kramer, M. Gastpar and P. Gupta. Cooperative Strategies and Capacity Theorems for Relay Networks. IEEE Trans. Inf. Theory, 51(9):3037-3063, Sep. 2005.

[4] B. Schein and R. G. Gallager. The Gaussian parallel relay network. In Proc IEEE Int. Symp. Inf. Theory, Sorrento, Italy, Jun. 2000, p.22.

[5] L. L. Xie and P. R. Kumar. An achievable rate for the multiple-level relay channel. IEEE Trans. Inf. Theory, 51(4):1348-1358, Apr. 2005.

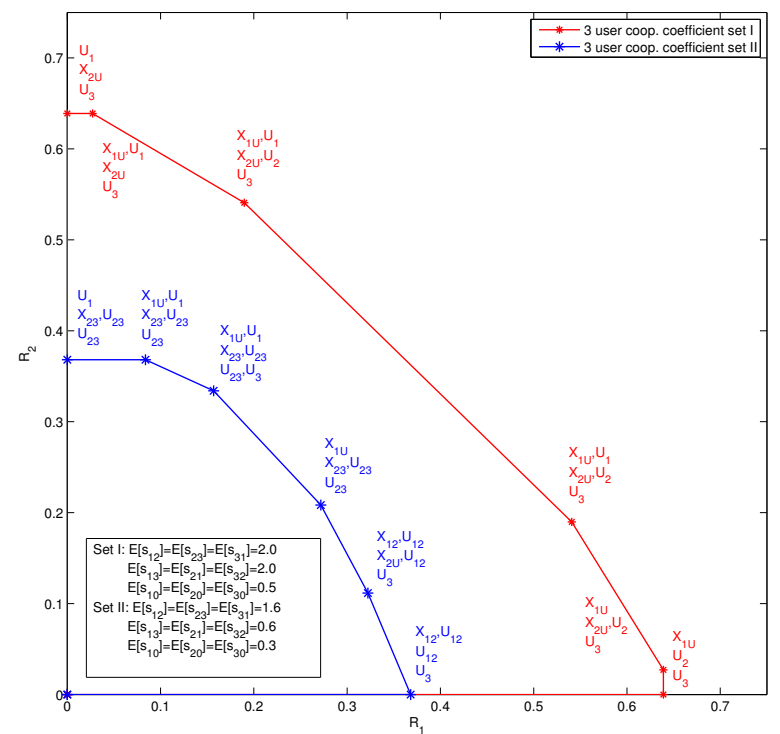

Fig. 3: Demonstration of active codewords in three user cooperation under symmetric vs asymmetric fading.

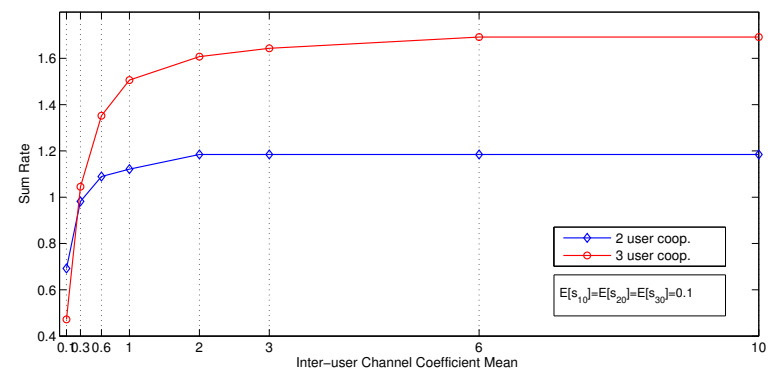

Fig. 4: Comparison of sum rates achievable by cooperation within two user and three user partitions of a multiuser system, with fixed total resources (power and bandwidth).

[6] L. Sankar, G. Kramer, and N. B. Mandayam. Offset Encoding for Multiple-Access Relay Channels. IEEE Trans. Inf. Theory, 53(10):38143821, Oct. 2007.

[7] A. Reznik, S.R. Kulkarni, and S. Verdu. Broadcast-relay channel: capacity region bounds. In Proc. IEEE Int. Symp. on Inf. Theory, Adelaide, Australia, Sep. 2005, pp: 820-824.

[8] J. N. Laneman and G. Wornell. Distributed Space-Time-Coded Protocols for Exploiting Cooperative Diversity in Wireless Networks. IEEE Trans. Inf. Theory, vol. 49, no. 10, pp. 2415-2425, Dec. 2004.

[9] A. B. Carleial. Multiple Access Channels with Different Generalized Feedback Signals. IEEE Trans. Inf. Theory, 28(6):841-850, Nov. 1982.

[10] F. M. J. Willems, E. C. van der Meulen and J. P. M. Schalkwijk. An Achievable Rate Region for the Multiple Access Channel with Generalized Feedback. In Proc. Allerton Conference, Monticello, IL, Oct. 1983.

[11] A. Sendonaris, E. Erkip and B. Aazhang. User Cooperation Diversity - Part I: System Description. IEEE Trans. on Communications, 51(11): 1927-1938, Nov. 2003.

[12] C. Edemen and O. Kaya, Achievable Rates for the Three User Cooperative Multiple Access Channel. In Proc. IEEE Wireless Communications and Networking Conference, Las Vegas, NV, Apr. 2008, pp: 1507-1512.

[13] C. Edemen and O. Kaya, Channel Adaptive Encoding and Decoding Strategies and Rate Regions for the Three User Cooperative Multiple Access Channel. In Proc. IEEE Globecom, New Orleans, LA, Dec. 2008, pp: $1-5$.

[14] M. A. Wigger and G. Kramer, Three-User MIMO MACs with Cooperation. In Proc. ITW 2009, Volos, Greece, June 10-12, 2009.

[15] O. Kaya and S. Ulukus. Power Control for Fading Cooperative Multiple Access Channels. IEEE Trans. on Wireless Communications, 6(8):29152923, Aug. 2007.

[16] T. Cover and J. Thomas. Elements of Information Theory. New-York: Wiley, 1991 